\title{
SÍNTESE, CARACTERIZAÇÃO E DEGRADAÇÃO DE P(3HB) POR Cupriavidus necator UTILIZANDO GLICEROL COMO SUBSTRATO
}

\author{
G.P. APATI ${ }^{1,2}$, A.L.S. SCHNEIDER ${ }^{1}$, B.R. SOMBRIO ${ }^{1}$, M.C.F. GARCIA ${ }^{1}$, A.P.T. PEZZIN ${ }^{1}$, M. \\ KELBERT $^{1,2}$ e A. FURIGO JUNIOR ${ }^{2}$ \\ ${ }^{1}$ Universidade da Região de Joinville - UNIVILLE, Departamento de Engenharia Química \\ ${ }^{2}$ Universidade Federal de Santa Catarina - UFSC, Departamento de Engenharia Química e Engenharia \\ de Alimentos \\ E-mail para contato: giannini.apati@ univille.br
}

\begin{abstract}
RESUMO - O poli(3-hidroxibutirato) - $\mathrm{P}(3 \mathrm{HB})$, poliéster natural e biodegradável, é considerado um atrativo substituto para os polímeros petroquímicos. $\mathrm{O}$ glicerol, que teve sua disponibilidade no mercado ampliada em decorrência da política nacional de estímulo à adição de biodiesel nos combustíveis fósseis, pode ser utilizado para diversas finalidades, dentre elas, como fonte de carbono no cultivo de micro-organismos. Assim, objetivou-se neste trabalho utilizar glicerol como fonte de carbono adicional na produção de $\mathrm{P}(3 \mathrm{HB})$. Foram realizados ensaios em biorreator com avaliação cinética do cultivo e extração dos filmes para a sua caracterização físico-química, térmica e mecânica. E, finalmente, foram realizados os ensaios de biodegradação em solo e exposição em câmara de envelhecimento acelerado, com avaliação do material do material degradado. verificou-se que o uso do glicerol não modificou as propriedades dos filmes. Em solo, as amostra degradaram em 35 dias.
\end{abstract}

Palavras-chave: Cupriavidus necator; Polihidroxibutirato; Glicerol

\section{INTRODUÇÃO}

Os materiais plásticos são parte integral da vida contemporânea e têm sido usados intensivamente devido às suas características, tais como, durabilidade, facilidade de processamento e resistência à biodegradação. No entanto, os plásticos convencionais, tais como o polipropileno (PP), poliestireno (PS), polietileno (PE) e poli (cloreto de vinila) (PVC) apresentam taxas extremamente baixas de degradação, o que pode levar a sérios problemas relativos ao desequilíbrio ambiental, como, por exemplo, às quantidades crescentes de resíduos plásticos que se acumulam na natureza (CHIELLINI e SOLARO, 1996). Em resposta a estes problemas e do efeito prejudicial da disposição destes plásticos no meio ambiente, vem aumentando consideravelmente o interesse pelo desenvolvimento dos materiais plásticos biodegradáveis, como os polihidroxialcanoatos (PHAs).

O poli(3-hidroxibutirato) ou $\mathrm{P}(3 \mathrm{HB})$, é um poliéster natural e biodegradável da família dos PHAs, sintetizado e acumulado no interior de diversos micro-organismos (ANDERSON e DAWES, 1990). Entretanto, o alto custo de produção dos PHAs tem limitado seu uso para a grande maioria das 


\section{9 a 22 de outubro de 2014 \\ Florianópolis/SC}

aplicações. Como cerca de $45 \%$ do custo total de produção pode ser atribuído à matéria prima, na qual a fonte de carbono é a mais consumida (CHOI e LEE, 1997), torna-se interessante o desenvolvimento de processos de produção baseados em fontes de carbono residuais. Por outro lado, o crescimento da produção de biodiesel, na qual o principal coproduto é o glicerol, tem gerado um excedente deste composto, sendo necessário buscar alternativas para sua destinação. Assim, uma alternativa interessante para utilização do glicerol é a sua bioconversão em produtos de alto valor agregado por meio de processos microbiológicos (SILVA et al., 2009).

De acordo com Gumel et al. (2012) o tipo da fonte de carbono utilizada como substrato não somente afeta o polímero produzido em termos das propriedades físico-químicas e mecânicas, como também seu rendimento e composição monomérica. Diante do exposto foi realizado um estudo da biossíntese de $\mathrm{P}(3 \mathrm{HB})$ por Cupriavidus necator utilizando glicerol como substrato adicional ao meio de cultivo avaliando suas propriedades físico-químicas, mecânicas, biodegradação em solo e fotodegradação.

\section{MATERIAL E MÉTODOS}

Neste trabalho cultivou-se a bactéria Cupriavidus necator DSM 545 em meio mineral formulado por Aragão et al. (1996). Os ensaios foram conduzidos em biorreator com cuba de 2 L (volume útil de 1,5 L) em duas condições: com e sem glicerol, utilizando glicerol purificado. As concentrações de açúcar invertido $\left(30\right.$ g. $\left.\mathrm{L}^{-1}\right)$ e glicerol $\left(15 \mathrm{~g} . \mathrm{L}^{-1}\right)$ e a temperatura de cultivo $\left(38^{\circ} \mathrm{C}\right)$ foram definidos por meio de um delineamento composto central rotacional (DCCR) $2^{3}$ completo com 3 pontos centrais (dados não apresentados). Os filmes obtidos a partir dos cultivos foram preparados pela técnica de evaporação lenta em solvente (clorofórmio) e submetidos aos ensaios de biodegradação em solo. Para tanto, o polímero foi dissolvido em clorofórmio na razão de $1 \%(\mathrm{~m} / \mathrm{V})$ em recipiente fechado, sendo aquecido (máximo de $80{ }^{\circ} \mathrm{C}$ ) sob agitação magnética. Após a total dissolução, verteu-se essa solução em n-hexano na proporção de 1:2 (V:V), sem aquecimento, mas sob constante agitação. Imediatamente foram formados cristais poliméricos, recuperados por filtração a vácuo. $\mathrm{O}$ produto seco retido na membrana foi pesado novamente e dissolvido em clorofórmio na concentração de $0,1 \mathrm{~g} \mathrm{~L}^{-1}$, vertido sobre uma placa de Petri e deixado evaporar para formação do filme em uma cuba de vidro onde o solvente foi eliminado em atmosfera saturada de clorofórmio. Para os ensaios de biodegradação foram utilizados copos de Becker de $17 \mathrm{~cm}$ contendo a amostra cortada no tamanho $5 \mathrm{~cm}^{2}$. As amostras foram acondicionadas em sacos de nylon para não serem perdidas no solo e possibilitar a análise da perda de massa durante a degradação. As amostras foram removidas após 7, 14, 21, 28 e 35 dias, lavadas em água destilada e secas em estufa a vácuo a $40{ }^{\circ} \mathrm{C}$ por $24 \mathrm{~h}$. Foram avaliadas mudanças por meio das mudanças morfológicas (avaliação visual e MEV), perda de massa, das propriedades térmicas (TGA e DSC) e variação do grau de cristalinidade (DRX). As amostras foram também submetidas a fotodegradação em câmara de envelhecimento acelerado e foram avaliadas periodicamente por mais de 90 dias. Os filmes obtidos com tamanhos de 50 × $1 \mathrm{~mm}$ foram expostos em uma câmara de envelhecimento acelerado, de acordo com a norma ASTM G154-06, mantidas a temperatura média de $45^{\circ} \mathrm{C}$, umidade do ar em $65 \%$, sob incidência direta 
dos raios de lâmpadas UV. O equipamento possui oito lâmpadas de mercúrio fluorescentes (Cleo Desempenho $80 \mathrm{~W}$ - PHILIPS), UVA $=22 \mathrm{~W}$ e UVA / UVB $=0,8 \%$. A fonte de radiação foi de lâmpadas fluorescentes Philips de $80 \mathrm{~W}$, com irradiação de $310 \mathrm{~nm}$ e $400 \mathrm{~nm}$, com uma média de radiação de cerca de $340 \mathrm{~nm}$, com boa equivalência com a radiação solar. Sob as condições utilizadas a dose diária de radiação ultravioleta é de cerca de $0,77 \mathrm{~W} / \mathrm{m} / \mathrm{nm} \mathrm{Wh} / \mathrm{m} 2$.

As amostras foram retiradas aos 7, 14, 28, 49, 63, 77 e 91 dias de exposição e pesadas a fim de verificar a redução de massa durante a degradação pela irradiação UV.

\section{RESULTADOS E DISCUSSÃO}

$\mathrm{Na}$ biossíntese a produção e o acúmulo de $\mathrm{P}(3 \mathrm{HB})$ no interior da célula foram muito similares 9,1 g /L e 56,1 \% na presença de glicerol puro no meio e 9,4 g/L e 59,0 \% quando não havia glicerol. Na Figura 1, está ilustrada a cinética do cultivo realizado em meio contendo $30 \mathrm{~g} \mathrm{~L}^{-1}$ de açúcar invertido e $15 \mathrm{~g} \mathrm{~L}^{-1}$ de glicerol purificado (AI/GP). Na Figura 2, está apresentada a cinética do cultivo realizado em meio com $30 \mathrm{~g} \mathrm{~L}^{-1}$ de açúcar invertido (AI/SG). Quando glicerol bruto proveniente da manufatura de biodiesel foi utilizado como cosubstrato, a produção de $\mathrm{P}(3 \mathrm{HB})$ foi extremamente baixa $0,4 \mathrm{~g} / \mathrm{L}$ para glicerol obtido tanto pela rota etílica quanto pela rota metílica.

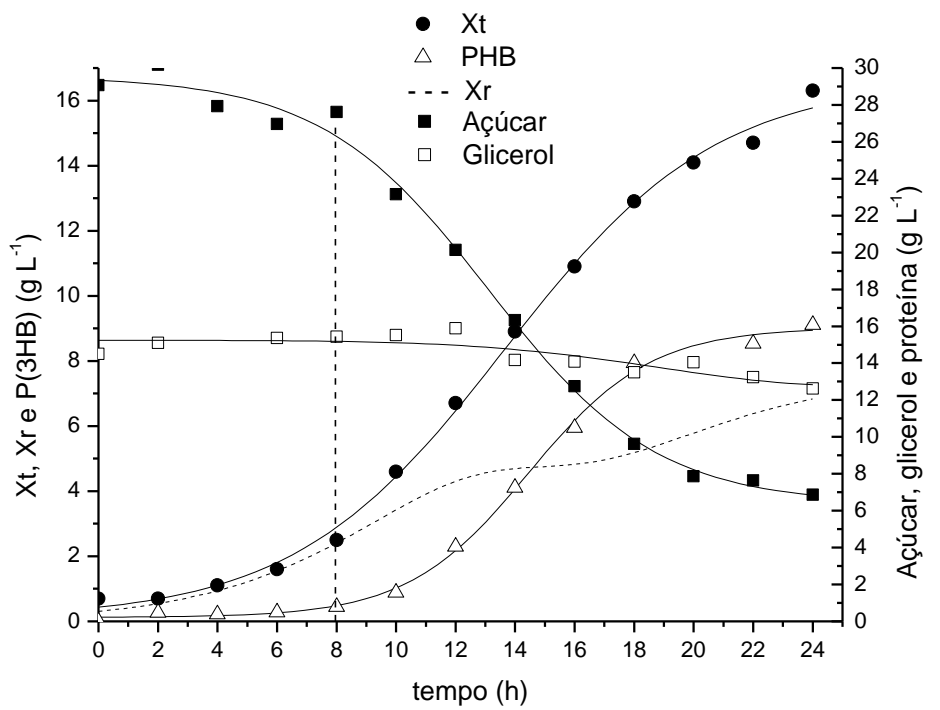

Figura 1. Cinética de crescimento de Cupriavidus necator em biorreator e produção de $\mathrm{P}(3 \mathrm{HB})$ a $38^{\circ} \mathrm{C}$, utilizando $30 \mathrm{~g} \mathrm{~L}^{-1}$ de açúcar invertido e $15 \mathrm{~g} \mathrm{~L}^{-1}$ de glicerol como substrato (AI/GP). ( ) biomassa total (Xt), $(\triangle)$ produção de $\mathrm{P}(3 \mathrm{HB})$, (---) biomassa residual (Xr), (ם) concentração de açúcar invertido (Açúcar), ( $\square$ ) concentração de glicerol. 


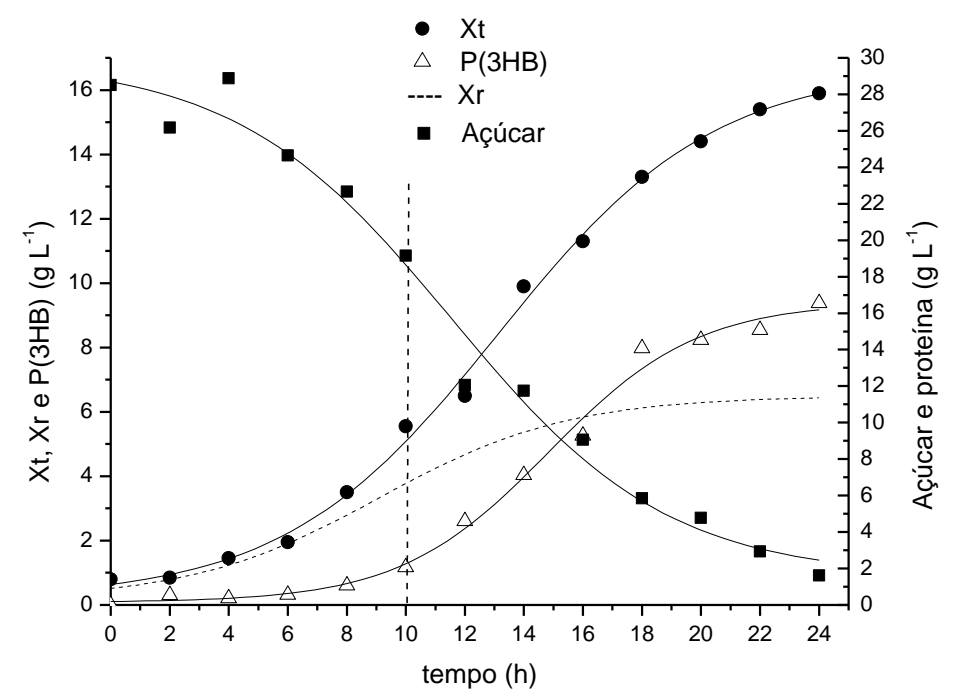

Figura 2. Cinética de crescimento de Cupriavidus necator em biorreator e produção de $\mathrm{P}(3 \mathrm{HB})$ a $38^{\circ} \mathrm{C}$, utilizando $30 \mathrm{~g} \mathrm{~L}^{-1}$ de açúcar invertido como substrato (AI/SG). $(\bullet)$ biomassa total $(\mathrm{Xt}),(\triangle)$ produção de P(3HB), (---) biomassa residual (Xr), ( $\square$ ) concentração de açúcar invertido (Açúcar), ( $\square$ ) concentração de glicerol.

Os filmes poliméricos foram caracterizados pelas técnicas de espectroscopia no infravermelho com transformada de Fourier (FTIR), difratometria de raios-X (DRX), análise termogravimétrica (TGA), calorimetria exploratória diferencial (DSC), ensaios mecânicos sob tensão e microscopia eletrônica de varredura (MEV). Na Tabela 1 estão apresentados um resumo das propriedade do filmes obtidos com e sem glicerol no meio de cultivo.

Tabela 1. Características físico-químicas e térmicas dos filmes de $\mathrm{P}(3 \mathrm{HB})$ sintetizados com e sem glicerol no meio.

\begin{tabular}{|c|c|c|c|c|c|c|c|c|}
\hline Amostra & $\begin{array}{l}\mathbf{T}_{\text {onset }} \\
\left({ }^{\circ} \mathbf{C}\right)\end{array}$ & $\begin{array}{c}\% \text { mass } \\
\text { loss }\end{array}$ & $\mathbf{T}_{\max }$ & $\begin{array}{c}\alpha c-D R X \\
(\%)\end{array}$ & $\mathbf{T}_{\mathrm{g}}\left({ }^{\circ} \mathbf{C}\right)$ & $\mathbf{T}_{\mathrm{m}}\left({ }^{\mathbf{o}} \mathbf{C}\right)$ & $\Delta \mathbf{H}_{\mathrm{m}}(\mathbf{J} / \mathbf{g})$ & $\begin{array}{c}\alpha c-D S C \\
(\%)\end{array}$ \\
\hline $\begin{array}{l}\mathrm{P}(3 \mathrm{HB})-\mathrm{com} \\
\text { glicerol }\end{array}$ & 242,7 & 98,5 & 254,2 & 60,7 & $-3,3$ & $\begin{array}{c}161,2 \mathrm{e} \\
176,4\end{array}$ & 88,7 & 60,8 \\
\hline $\begin{array}{l}\mathrm{P}(3 \mathrm{HB})-\text { sem } \\
\text { glicerol }\end{array}$ & 242,8 & 99,4 & 256,3 & 61,6 & 5,7 & $\begin{array}{c}162,6 \mathrm{e} \\
177,0\end{array}$ & 90,0 & 61,6 \\
\hline
\end{tabular}

Quanto às propriedades mecânicos os dois filmes apresentaram características bem semelhantes entre si. O módulo de Young foi de 2,46 \pm 0,98 GPa para o $\mathrm{P}(3 \mathrm{HB})$ com glicerol e 2,09 \pm 0,39 $\mathrm{GPa}$ para o $\mathrm{P}(3 \mathrm{HB})$ sem glicerol. $\mathrm{O}$ alongamento na ruptura encontrado foi $1,19 \pm 0,09 \%$ para o $\mathrm{P}(3 \mathrm{HB})$ sintetizado com glicerol e $1,17 \pm 0,26 \%$ para o $\mathrm{P}(3 \mathrm{HB})$ sem glicerol, mostrando que o polímero deforma muito pouco antes de romper. Os valores de tensão máxima obtidos foram 24,05 \pm 8,10 MPa e 19,12 $\pm 5,84 \mathrm{MPa}$ para os polímeros com glicerol e sem glicerol, respectivamente. 


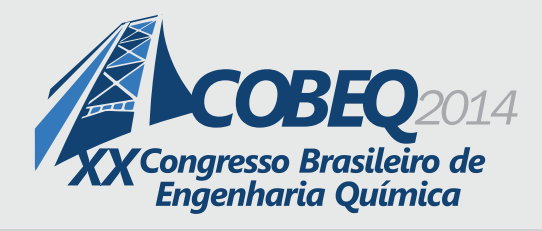

19 a 22 de outubro de 2014
Florianópolis/SC

A morfologia dos filmes obtidos nos bioprocessos com e sem glicerol foram observadas por MEV (Figura 3). A partir de 14 dias já é possível observar sinais claros de que as amostras começaram a ser degradadas. A partir de 21 dias as amostras já estão claramente deterioradas sendo possível observar trincas e diversos orifícios e fragmentação das amostras, e após 35 dias não foi mais encontrada amostra, sendo que neste tempo já estava totalmente biodegradada. Na exposição dos filmes em câmara de envelhecimento acelerado houve perda na massa dos polímeros totalizando 2,4 e $3,7 \%$, sintetizados com e sem glicerol no meio, respectivamente, no decorrer de mais de 90 dias de ensaio, podendo considerar que esses polímeros são estáveis para usos diversos, sendo degradados somente após seu descarte.
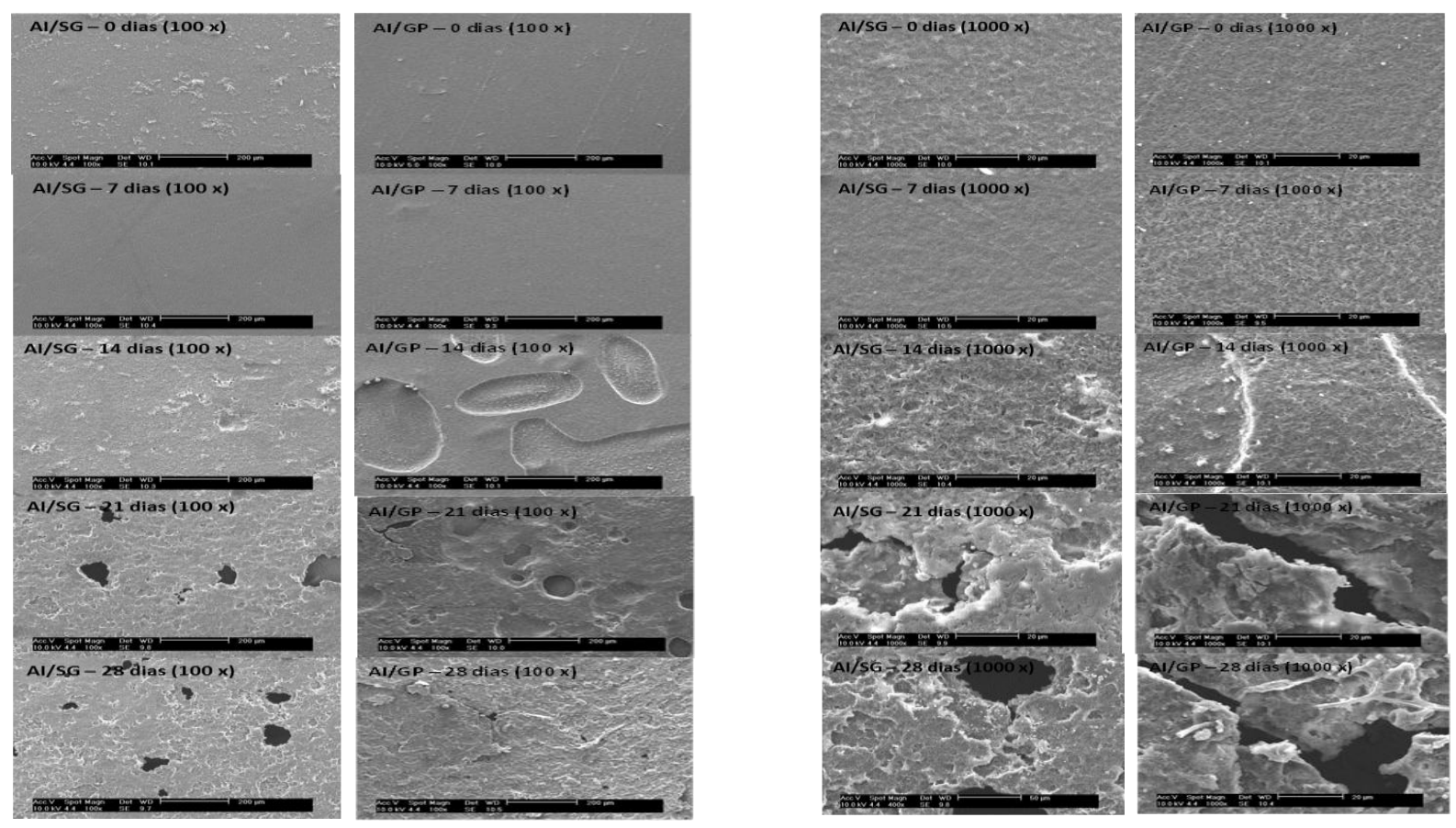

Figura 3. Micrografias de MEV (100x e 1000x) das amostras AI/SG e AI/GP após 0, 7, 14, 21 e 28 dias de biodegradação em solo.

\section{CONCLUSÃO}

$\mathrm{Na}$ caracterização dos polímeros sintetizados em biorreator com e sem adição de glicerol puro, verificou-se que o uso do glicerol não modificou as propriedades dos filmes. Em solo, as amostra degradaram em 35 dias, enquanto na exposição dos filmes à câmara de envelhecimento acelerado notou-se maior estabilidade das amostras o que permitiria a aplicação do polímero no ambiente, sem que ele se degradasse. 


\section{REFERÊNCIAS}

ANDERSON A.J.; DAWES, E.A. Microbiological Reviews. v.54, p. 450-472, 1990.

ARAGÃO, G. M. F.; LINDLEY, N. D.; URIBELARREA, J. L.; PAREILLEUX, Biotechnology Letters. v.18, p.937-942, 1996.

CHIELLINI, E; SOLARO, R. Biodegradable polymeric materials. Advanced Materials. 8, n 4, 1996.

CHOI, J.; LEE, S.Y. Process analysis and economic evaluation for poly(3-hydroxybutyrate) production by fermentation. Bioprocess Engineering. v.17, p.335-342, 1997.

GUMEL, A.M; ANNUA, M.S.M; HEIDELBERG, T. Polym. Degrad. and Stability. v.97, p.12241231, 2012.

SILVA, G.P.; MACK, M.; CONTIERO, J. Biotecnol. Advances. v.27, p.30-39, 2009. 